Service social

Psychiatrie clinique, approche bio-psycho-sociale, par Pierre Lalonde, Frédéric Grunberg et collaborateurs, Gaëtan Morin éditeur, 2e édition, 1988, 1348 pages (distribué en Europe et en Afrique par les éditions Eska).

\title{
Marie Émond
}

Volume 39, numéro 2, 1990

Les problèmes sociaux

URI : https://id.erudit.org/iderudit/706496ar

DOI : https://doi.org/10.7202/706496ar

Aller au sommaire du numéro

Éditeur(s)

École de service social de l'Université Laval

ISSN

1708-1734 (numérique)

Découvrir la revue

Citer ce compte rendu

Émond, M. (1990). Compte rendu de [Psychiatrie clinique, approche

bio-psycho-sociale, par Pierre Lalonde, Frédéric Grunberg et collaborateurs,

Gaëtan Morin éditeur, 2e édition, 1988, 1348 pages (distribué en Europe et en Afrique par les éditions Eska).] Service social, 39(2), 254-255.

https://doi.org/10.7202/706496ar d'utilisation que vous pouvez consulter en ligne. 
vers le psychologique et du didactique vers le ludique » (p. 73). Plus important encore, on y effectue un retour sur la notion du chômage créateur et on y propose des perspectives de relance, avec des vues précises par rapport à certains paramètres tels que la clientèle, les objectifs d'intervention, le milieu d'intervention, le cadre d'intervention, l'équipe d'intervention, le contenu du programme, etc. Certains lieux communs y sont enfin démystifiés, tels le développement de "l'employabilité " et l'incitation à la création d'entreprises. Un bilan donc d'une belle facture et d'une grande qualité. Je n'aurais qu'un reproche à lui adresser: que la dynamique de la relation vécue entre intervenants-intervenantes et participants-participantes ait été délibérément mise de côté (p. 52-53). C'est la dimension qu'il resterait à explorer pour une vue complète de la relance à effectuer.

Une version abrégée de ce rapport d'évaluation est disponible sous la description bibliographique suivante : Vivre malgré l'absence d'emploi : le chômage créateur, par Michel Boyer, Marie-Chantal Guédon et Anne Kettenbeil, Saint-Jean-sur-Richelieu, Centre de formation à l'Autogestion du HautRichelieu, 1989, 24 pages. Je recommande la lecture de la version intégrale pour une pleine compréhension de la nature et de la portée de l'intervention réalisée.

Gérald Doré

École de service social

Université Laval

\section{Psychiatrie clinique, approche bio-psycho-sociale, par Pierre LALONDE, Frédéric GRUNBERG et collaborateurs, Gaëtan Morin éditeur, $2^{\mathrm{e}}$ édition, 1988, 1348 pages (distribué en Europe et en Afrique par les éditions Eska).}

"Une psychiatrie qui continue d'être française et européenne tout en s'inspirant au maximum des travaux des écoles américaines "nous dit-on dans la préface. II s'agit d'une tentative intéressante de synthèse, à partir de notre situation géographique et politique particulière, et d'intégration des théories multiples qui influencent la compréhension des comportements humains, normaux et psychopathologiques.

Ce livre est clair et précis, de lecture agréable et facile. II faut rendre hommage à ce souci de clarté et à l'évitement systématique d'un vocabulaire obscur et hermétique. L'impression est nette, la mise en page soignée et variée, le tout bien aéré. Un produit final qui met en appétit de lecture !

L'ouvrage fait appel à cinquante-quatre collaborateurs et est divisé en six parties. On y traite des sujets suivants : introduction à la psychiatrie, état actuel de la psychiatrie, relation médecin et malade, épidémiologie, psychophysiologie, développement de la personnalité, examen psychiatrique, syndromes cliniques psychiatriques (des troubles anxieux, en passant par l'alcoolisme et les urgences), troubles de la sexualité, aspects spécifiques de la psychiatrie (femme-famille-couple), pédopsychiatrie (de l'évaluation aux stades de développement) et enfin, traitements psychiatriques divisés en trois parties : traitements pharmacologiques, psychothérapies et thérapies psychophysiologiques. Une dernière partie aborde plus spécifiquement les aspects administratifs et légaux. 
Faire un manuel n'est pas chose facile. Si celui-ci a quelque faiblesse, ce serait dans la sur-simplification de certains chapitres, notamment ceux traitant des aspects sociaux tels le couple, la famille, la femme, où on aurait eu intérêt à s'adjoindre des spécialistes d'autres disciplines afin de ramener les concepts utilisés au niveau du discours contemporain.

Nous avons mentionné les nombreux collaborateurs. Si cela fait un ouvrage très vivant et varié, il est cependant plus difficile d'arriver à une unité de ton et de qualité de contenu.

Si la plupart des chapitres sont tout à fait remarquables de concision, de rigueur, de richesse d'information, certains laissent à désirer dans l'organisation du matériel, dans le parti pris pour certaines approches sans justification pour le lecteur, dans des citations de recherches acceptées d'emblée après une présentation trop sommaire. À certains endroits, on ne cite que des auteurs américains, à d'autres, que des européens. L'intégration des trois pôles américain, québécois et européen, reste donc fragmentaire. Par moments, on retrouve des tableaux de référence avec adresses pour l'ensemble du Québec; ailleurs, ils sont absents. Certaines des bibliographies sont très longues. Des notes de lecture, qui s'avèrent une bonne idée, n'apparaissent que trop rarement. En somme la logique de certains détails semble dépendre davantage de chaque auteur que d'un souci d'unité.

Plus cocasses sont les petites guerres idéologiques! Par exemple autour de la psychanalyse, abondamment décriée par certains, abondamment citée par d'autres. Encore une fois l'unité de ton de l'ensemble en prend pour son rhume ! Et peut devenir une source de confusion pour le premier public visé qui est l'étudiant.

Enfin, grande absente du dernier chapitre, la multidisciplinarité du milieu soignant dans le travail quotidien en psychiatrie; celle-ci avec les problèmes qu'elle peut comporter, est pourtant un élément clé de l'intervention. Les structures de soins n'y sont pas non plus décrites autrement que dans l'organigramme d'ensemble. Il me semble qu'il y aurait lieu de compléter l'excellente description de l'évolution des services par certaines données sur l'opérationnalisation quotidienne des soins et par des statistiques sur la maladie mentale.

À lire, pour comprendre la définition actuelle du bio-psycho-social, pour votre culture générale, pour rafraîchir, acquérir ou augmenter votre propre vocabulaire et vos connaissances en psychiatrie, et pour démystifier le jargon psychiatrique.

Marie Émond

Clinique de pédopsychiatrie Pavillon Albert-Prévost Montréal 